\title{
On the Possibility of Supertasks in General Relativity
}

\author{
John Byron Manchak
}

\begin{abstract}
Malament-Hogarth spacetimes are the sort of models within general relativity that seem to allow for the possibility of supertasks. There are various ways in which these spacetimes might be considered physically problematic. Here, we examine these criticisms and investigate the prospect of escaping them.
\end{abstract}

\section{Introduction}

Within general relativity, some models seem to allow for the completion of an infinite number of tasks (a supertask) in finite amount of time. ${ }^{1}$ Such models - called Malament-Hogarth (M-H) spacetimes - may have significant implications for the foundations of computability theory. For example, some have argued that the supertasks enabled by $\mathrm{M}-\mathrm{H}$ spacetimes refute the physical Church's Thesis by allowing for the computation of a Turing non-computable function [11]. M-H spacetimes can be defined as follows [10].

Definition. We say $\left(M, g_{a b}\right)$ is an $M-H$ spacetime if there is a future-directed timelike half-curve $\gamma \subset M$ and a point $p \in M$ such that $\int_{\gamma} d \tau=\infty$ and $\gamma \subset I^{-}(p)$.

Here, the curve $\gamma$ represents the worldline of some observer. Because $\gamma$ has infinite proper time, she may complete an infinite number of tasks. But,

\footnotetext{
${ }^{1}$ What we mean by an "infinite number of tasks" is left deliberately vague; it is notoriously difficult to be careful about such things. An excellent introduction to supertasks as well as a survey of the relevant literature is given in [4]. For the background structure of general relativity, see [9] and [16].
} 
at every point in $\gamma$, it is possible to send a signal to the point $p$. Because there always exists a curve $\gamma^{\prime}$ with future endpoint $p$ which has finite proper time, we can think of $\gamma$ as the "sender" and $\gamma^{\prime}$ as the "receiver" of a signal. In this way, the receiver may complete an infinite number of tasks in a finite time. To illustrate these basic features of the definition, it helps to examine various instantiations. Here is a toy example [3]:

Example. Let $\left(\mathbb{R}^{2}, \eta_{a b}\right)$ be two-dimensional Minkwski spacetime. Consider a compact set $C \subset \mathbb{R}^{2}$, a point $q \in C$, and point $p$ such that $C \subset I^{-}(p)$. Now let $\Omega$ be a strictly positive smooth function on $\mathbb{R}^{2}-\{q\}$ such that, $\Omega=1$ outside of $C$ and, as $q$ is approached, $\Omega$ becomes infinite. $\left(\mathbb{R}^{2}-\{q\}, \Omega^{2} \eta_{a b}\right)$ is an $\mathrm{M}-\mathrm{H}$ spacetime with any future-directed timelike curve $\gamma$ approaching $q$ representing the sender and $p$ as a point on the worldine of the receiver where $\gamma \subset I^{-}(p)$ (see Figure 1).



Figure 1: The spacetime $\left(\mathbb{R}^{2}-\{q\}, \Omega^{2} \eta_{a b}\right)$.

Now we want to consider physical constraints that may be used to evaluate whether there exist M-H spacetimes in which a supertask may be plausibly completed. We want to assess not only the physical reasonableness of the M-H spacetime in question, but also the physical implementability of the supertask within that spacetime. To do this, we will need a way to represent 
such setups within M-H spacetimes. Consider the following definition.

Definition. Let $\left(M, g_{a b}\right)$ be an M-H spacetime. Let $\gamma \subset M$ be a futuredirected timelike half-curve such that $\int_{\gamma} d \tau=\infty$. Let $p \in M$ be such that $\gamma \subset I^{-}(p)$. We say the ordered triple $\left(\left(M, g_{a b}\right), p, \gamma\right)$ is an $M-H$ setup.

We note that there is, associated with every $\mathrm{M}-\mathrm{H}$ spacetime, an infinite number of $\mathrm{M}-\mathrm{H}$ setups. What follows are characterizations of certain conditions, many of which are found in [3], that an $\mathrm{M}-\mathrm{H}$ setup $\left(\left(M, g_{a b}\right), p, \gamma\right)$ must satisfy in order for it to be considered physically reasonable.

\section{Conditions of Physical Reasonableness}

The first condition, inextendibility, is one which is regularly used to rule out seemingly artificial models. Roughly, the idea is to require that spacetime extend out "as far as it can." Formally, we have the following.

Definition. A spacetime $\left(M, g_{a b}\right)$ is inextendible if there is no isometric embedding $\theta: M \rightarrow M^{\prime}$ into a spacetime $\left(M^{\prime}, g_{a b}^{\prime}\right)$ such that $M$ is a proper subset of $M^{\prime}$.

Though the metaphysics involved can be questioned, we will follow general practice and assume that, for any spacetime to be physically reasonable, it must be inextendible. ${ }^{2}$

Next, consider the following $\mathrm{M}-\mathrm{H}$ setup.

Example. Let $\left(\mathbb{S} \times \mathbb{R}, \eta_{a b}\right)$ be two-dimensional Minkowski spacetime which has been "rolled up" along the time axis. Let $\gamma$ be any future-inextendible timelike curve and let $p$ be any point in $\mathbb{S} \times \mathbb{R}$. The triple $\left(\left(\mathbb{S} \times \mathbb{R}, \eta_{a b}\right), p, \gamma\right)$ is an $\mathrm{M}-\mathrm{H}$ setup because $I^{-}(p)$ covers the entire manifold (see Figure 2).

Given this example, it may seem that $\mathrm{M}-\mathrm{H}$ setups are too easily constructed if causal misbehavior is permitted. This is the first worry noted by

\footnotetext{
${ }^{2}$ See [2, pp. 31-33] for a disucssion.
} 


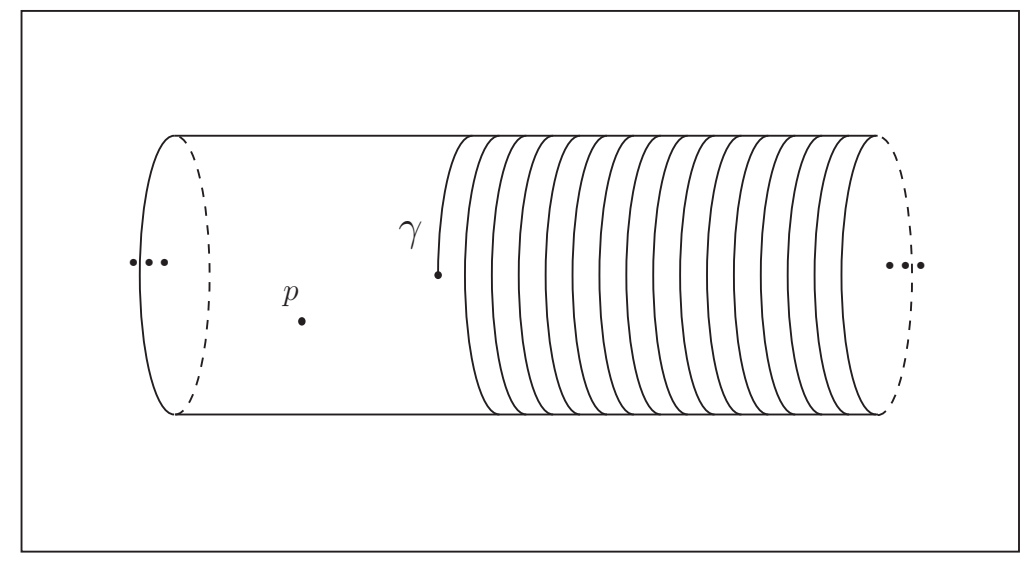

Figure 2: "Rolled" Minkowski spacetime.

Earman and Norton [3] and the intuition can be realized in the following easily proved proposition.

Proposition. If a spacetime contains closed timelike curves, then it is an M-H spacetime.

Proof. Let $\left(M, g_{a b}\right)$ be a spacetime. Let $\gamma \subset M$ be a closed timelike curve. Let $\gamma_{\infty} \subset M$ be the curve that results from staring at a point $p \in \gamma$ and looping around $\gamma$ an infinite number of times. Clearly $\gamma_{\infty}$ is, by construction, a timelike half-curve such that $\int_{\gamma_{\infty}} d \tau=\infty$. Of course, $\gamma_{\infty} \subset I^{-}(p)$. So $\left(M, g_{a b}\right)$ is an $\mathrm{M}-\mathrm{H}$ spacetime.

Whether acausal spacetimes should be classified as physically unreasonable is the topic of another paper. But for the time being, we can nonetheless require that $\mathrm{M}-\mathrm{H}$ spacetimes have well-behaved causal structure. There is one caveat, however. We cannot become too causally strict or we risk labeling all M-H spacetimes as physically unreasonable. We have the following result.

Proposition. (Malament) If a spacetime is globally hyperbolic, then it is not an M-H spacetime.

Proof. Given in [2, p. 122]. 
So, in order to entertain the the notion of M-H spacetimes, one must be willing to welcome at least a bit causal mischief. The task is to find some causal condition which is strong enough to exclude trivial M-H spacetimes but not too strong so as to eliminate all of them. Following [3], our preferred condition will be stable causality.

Definition. A spacetime $\left(M, g_{a b}\right)$ is stably causal if there exists a nonvanishing timelike vector field $\xi^{a}$ on $M$ such that the spacetime $\left(M, g_{a b}+\xi_{a} \xi_{b}\right)$ contains no closed timelike curves.

A second concern given in [3] has to do with possible infinite blueshifts recorded by the receiver. An informal argument summarizing the distress is recounted here:

During her lifetime, [the sender] measures an infinite number of vibrations of her source, each vibration taking the same amount of her proper time. [The receiver] must agree that an infinite number of vibrations take place. But within a finite amount of his proper time, [the receiver] receives an infinite number of light signals from [the sender], each announcing the completion of a vibration. For this to happen, [the receiver] must receive the signals in ever decreasing intervals of his proper time. Thus, [the receiver] will perceive the frequency of [the sender's] source to increase without bound [3, p. 30].

There is a technical result offered in support of the informal argument given above [3, p. 31]. The precise formulation of the result need not concern us here. For our purposes, it is enough to note that arbitrary M-H setups do not satisfy the antecedent assumptions of the given result. ${ }^{3}$ Thus, although the result nicely labels some M-H setups (those satisfying its assumptions) as blueshift delinquent, the lemma says nothing concerning setups falling outside its scope. Accordingly, we would like to find a condition that, if satisfied, would seem to label an M-H setup without blueshift complications as such.

\footnotetext{
${ }^{3}$ For example, it is not always possible to find a family of null geodesics connecting points in the the worldline of the sender to the worldline of the receiver. In addition, even assuming that such a family of null geodesics exists, there is no guarantee that they will form an integral submanifold or that the order of emission from the sender will match the order of reception by the receiver.
} 
We have the following.

Definition. Let $\left(\left(M, g_{a b}\right), p, \gamma\right)$ be an M-H setup. Let $\left\{q_{i}\right\}$ be a countably infinite sequence of points in $\gamma$ such that, for all $i$, the distance (as measured in proper time along $\gamma$ ) between $q_{i}$ and $q_{i+1}$ is the same. We say that $\left(\left(M, g_{a b}\right), p, \gamma\right)$ satisfies the bounded blueshift condition if (i) there exists a timelike curve $\gamma^{\prime}$ with future endpoint $p$ such that there is a future-directed null geodesic $K_{i}$ from each $q_{i}$ to some $q_{i}^{\prime} \in \gamma^{\prime}$ and (ii) there is a $\delta>0$ such that, for all $i$, the quantity $\left(V^{\prime} a k_{a}^{\prime}\right)_{\mid} q_{i}^{\prime} /\left(V^{a} k_{a}\right)_{\mid} q_{i}$ is less than $\delta$ where $V_{\mid q_{i}}^{a}$ and $V_{\mid q_{i}^{\prime}}^{\prime a}$ are, respectively, the unit tangent to $\gamma$ at $q_{i}$ and the unit tangent to $\gamma^{\prime}$ at $q_{i}^{\prime}$ and $k_{\mid q_{i}}^{a}$ and $k_{\mid q_{i}^{\prime}}^{a}$ are, respectively, the tangents to $K_{i}$ at $q_{i}$ and $q_{i}^{\prime}$.

We want to emphasize that it is not clear from the outset that any $\mathrm{M}-\mathrm{H}$ setups satisfy the bounded blueshift condition. In fact, citing their proposition and numerous examples, Earman and Norton seem to think that the divergent blueshift problem is essentially inescapable [3, p. 33].

Next, we consider a third worry given in [3]. Some spacetimes, such as the toy example mentioned above, do not satisfy the the so-called "energy conditions". For each spacetime $\left(M, g_{a b}\right)$, we associate via Einstein's equation, a stress-energy tensor $T_{a b}$ defined on all of $M$. The energy conditions are constraints on placed on $T_{a b}$ which are thought to be satisfied by all physically reasonable matter sources. We will define two of these energy conditions here. ${ }^{4}$

Definition. A spacetime $\left(M, g_{a b}\right)$ satisfies the dominant energy condition if, for all future directed timelike vectors $\xi^{a}$, then $T_{b}^{a} \xi^{b}$ is a future directed causal vector where $T_{a b}$ is the stress-energy tensor associated with $\left(M, g_{a b}\right)$.

Definition. A spacetime $\left(M, g_{a b}\right)$ satisfies the strong energy condition if, for all unit timelike vectors $\xi^{a}$, then $T_{a b} \xi^{a} \xi^{b} \geq-\frac{1}{2} T_{a}^{a}$ where $T_{a b}$ is the stressenergy tensor associated with $\left(M, g_{a b}\right)$.

Next, we note that in some M-H setups $\left(\left(M, g_{a b}\right), p, \gamma\right)$, the curve $\gamma$ is a

\footnotetext{
${ }^{4} \mathrm{~A}$ third energy condition, not defined here is called the "weak energy condition" and is satisfied whenever the dominant energy condition is. For more on the energy conditions, see [3].
} 
geodesic but that this is not always the case. Consider a setup generated from anti-de Sitter spacetime.

Example. Let $t$ and $x$ be standard coordinates on $\mathbb{R}^{2}$. Consider the spacetime $\left(\mathbb{R}^{2}, g_{a b}\right)$ where $g_{a b}=\cosh ^{2} x \nabla_{a} t \nabla_{b} t-\nabla_{a} x \nabla_{b} x$. Here, the null cones rapidly widen as $x$ increases in absolute value. Thus, for any point $p \in \mathbb{R}^{2}$, there are future-directed timelike curves $\gamma$ in $I^{-}(p)$ with infinite proper time (see Figure 3).

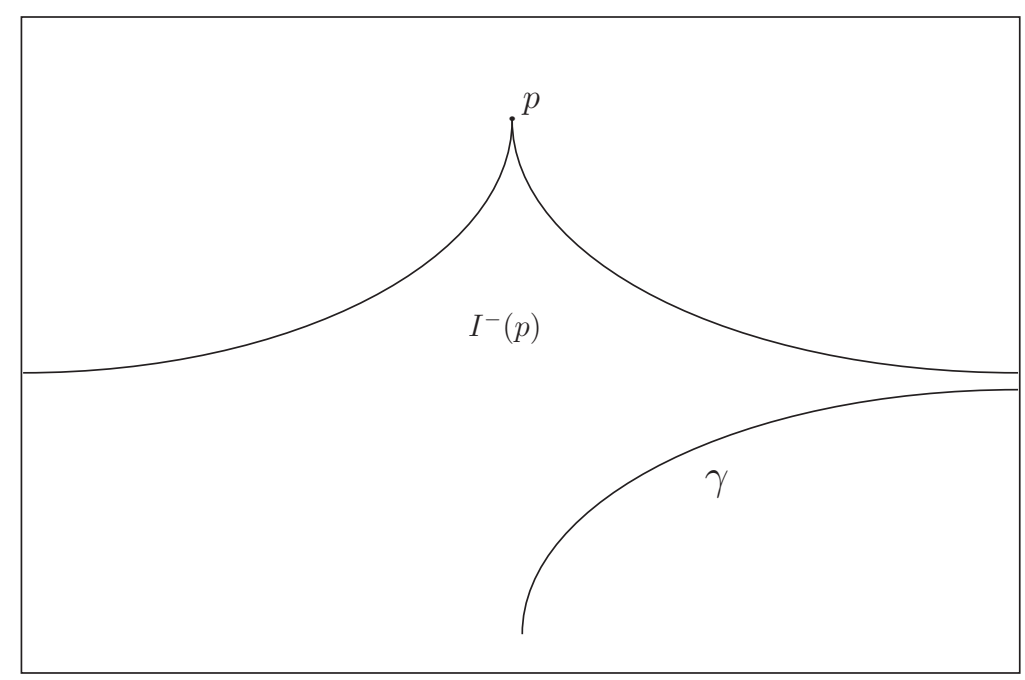

Figure 3: Anti-de Sitter spacetime.

In the example above, it can be shown that not only is $\gamma$ not a geodesic, but its total (integrated) acceleration is infinite [3, p. 36]. Given such examples, Earman and Norton demand a finite bound on the total acceleration of $\gamma$. This seems sensible - a rocket ship which carries a finite amount of fuel cannot traverse a curve with infinite total acceleration (assuming it cannot refuel along the way) [1]. So, we have the following.

Definition. We say that the M-H setup $\left(\left(M, g_{a b}\right), p, \gamma\right)$ satisfies the finite acceleration condition if $\int_{\gamma} a d \tau<\infty$ where $a$ is the scalar value of acceleration defined at each point in $\gamma$.

In an M-H setup $\left(\left(M, g_{a b}\right), p, \gamma\right)$, the sender $\gamma$ is represented by the world- 
line of a point particle. However, it has been emphasized that such representations are not realistic [3]. Instead of a single worldline $\gamma$, one must be concerned with a congruence $\Gamma$ of such worldlines which more appropriately represents the sender. But, if the sender is to carry out her tasks, we must verify that the tidal forces on such a congruence $\Gamma$ remain bounded. Associated with each spacetime $\left(M, g_{a b}\right)$ is a Riemann curvature tensor $R_{b c d}^{a}$ defined on all of $M$. Because the tidal forces are proportional to the Riemann curvature tensor, we feel the following constraint is a reasonable one to place on spacetime:

Definition. A spacetime $\left(M, g_{a b}\right)$ satisfies the tidal force condition if there exists a $k>0$ such that the Kretschmann curvature scalar $R_{a b c d} R^{a b c d}$ is less than $k$ and greater than $-k$ at all points in $M$.

Earman and Norton have a sixth worry. They contend that the receiver in an M-H setup can always question the reliability of the signal sent by the sender. The concern has its roots in the following precise claim [3, p. 37].

Claim. Let $\left(\left(M, g_{a b}\right), p, \gamma\right)$ be an M-H setup. If $\Sigma \subset M$ is a spacelike hypersurface such that $\gamma \subset I^{+}(\Sigma)$, then $p \notin \operatorname{int}\left[D^{+}(\Sigma)\right] .^{5}$

The idea is that $\Sigma$ is a spacelike surface from which the physical situation on all of $D^{+}(\Sigma)$ can be determined. If $\gamma$ were in $D^{+}(\Sigma)$, then initial conditions on $\Sigma$ would guarantee the completion of the sender's tasks and signals. Clearly, $\gamma \subset D^{+}(\Sigma)$ implies $\gamma \subset I^{+}(\Sigma)$. However, as Earman and Norton point out, if $p \notin \operatorname{int}\left[D^{+}(\Sigma)\right]$ then "events at $p$ or at points arbitrarily close to $p$ are subject to nondeterministic influences" [3, p. 38]. So, if the above claim is true, there would always be room for the receiver to doubt the reliability of the sender's signal. Earman and Norton have offered a proof of the claim so it would seem that the matter has been settled. However, we note here that the claim is false as presented. ${ }^{6}$

The formulation of the claim can be altered so as to make it true by assuming that $\Sigma$ is achronal; it is likely that this was the intended theorem. But we note that this additional assumption significantly weakens the result

\footnotetext{
${ }^{5}$ Here, as in [3, p. 35], we define the future domain of dependence $D^{+}(\Sigma)$ of $\Sigma$ as the set of all points $p \in M$ such that every causal curve which passes through $p$ without past endpoint meets $\Sigma$. This agrees with the formulation given in [9].

${ }^{6}$ For a counterexample, the interested reader may consult the proposition below.
} 
and we question its imposition.

In some texts, the future domain of dependence $D^{+}(\Sigma)$ of a set $\Sigma$ is only defined when $\Sigma$ is achronal [16]. But, we want to make it very clear that this is done for mathematical convenience only [5, p. 438]. The domain of dependence can be and, in places such as [3] and [9], has been defined for arbitrary sets. So, we emphasize that the achronality of $\Sigma$ is not implicit in the formulation of the claim above.

One attempt to justify the achronality assumption is to argue that nonachronal spacelike hypersurfaces somehow signal causal misbehavior. But such an argument is inadequate. While it is true that some causally delinquent spacetimes do not admit any achronal spacelike hypersufaces (e.g. Gödel spacetime), every spacetime has innumerable spacelike hypersurfaces which are not achronal. ${ }^{7}$. So the existence of such surfaces in no way flags causal mischief.

Besides, we argue that any worries concerning unruly causal structure should be formulated as a condition satisfied by the whole of spacetime. We have, for example, already stipulated that (for our purposes) an M-H spacetime must be stably causal to be considered physically reasonable. In our opinion, this seems to be enough to rule out causal pathologies.

Consequently, we feel that the claim above can be used to formulate a condition on $\mathrm{M}-\mathrm{H}$ setups ensuring signal reliability. We have the following.

Definition. The M-H setup $\left(\left(M, g_{a b}\right), p, \gamma\right)$ satisfies the signal reliability condition if there is a spacelike hypersurface $\Sigma \subset I^{-}(p)$ such that $\gamma \subset D^{+}(\Sigma)$ and $p \in \operatorname{int}\left[D^{+}(\Sigma)\right]$.

Here, we have required that not only $\gamma$ be in $I^{+}(\Sigma)$ but that it be in $D^{+}(\Sigma)$ as well. We have also insisted that $\Sigma$ be in $I^{-}(p)$. Together, these constraints ensure that the receiver at $p$ has the resources to determine the behavior of $\gamma$. In addition, we demand that $p \in \operatorname{int}\left[D^{+}(\Sigma)\right]$. This secures the determination, from $p$, of events sufficiently close to $p$. Hence, the reliability of the sender's signal is guaranteed.

Clearly, the rolled Minkowski spacetime example given above satisfies the signal reliability condition. However, it is not clear from the outset that the condition is satisfied by any causally well-behaved M-H setup. All examples considered in [3] do not. This includes Reissner-Nordström spacetime.

\footnotetext{
${ }^{7}$ For an example in Minkowski spacetime, see [8, p. 246]
} 


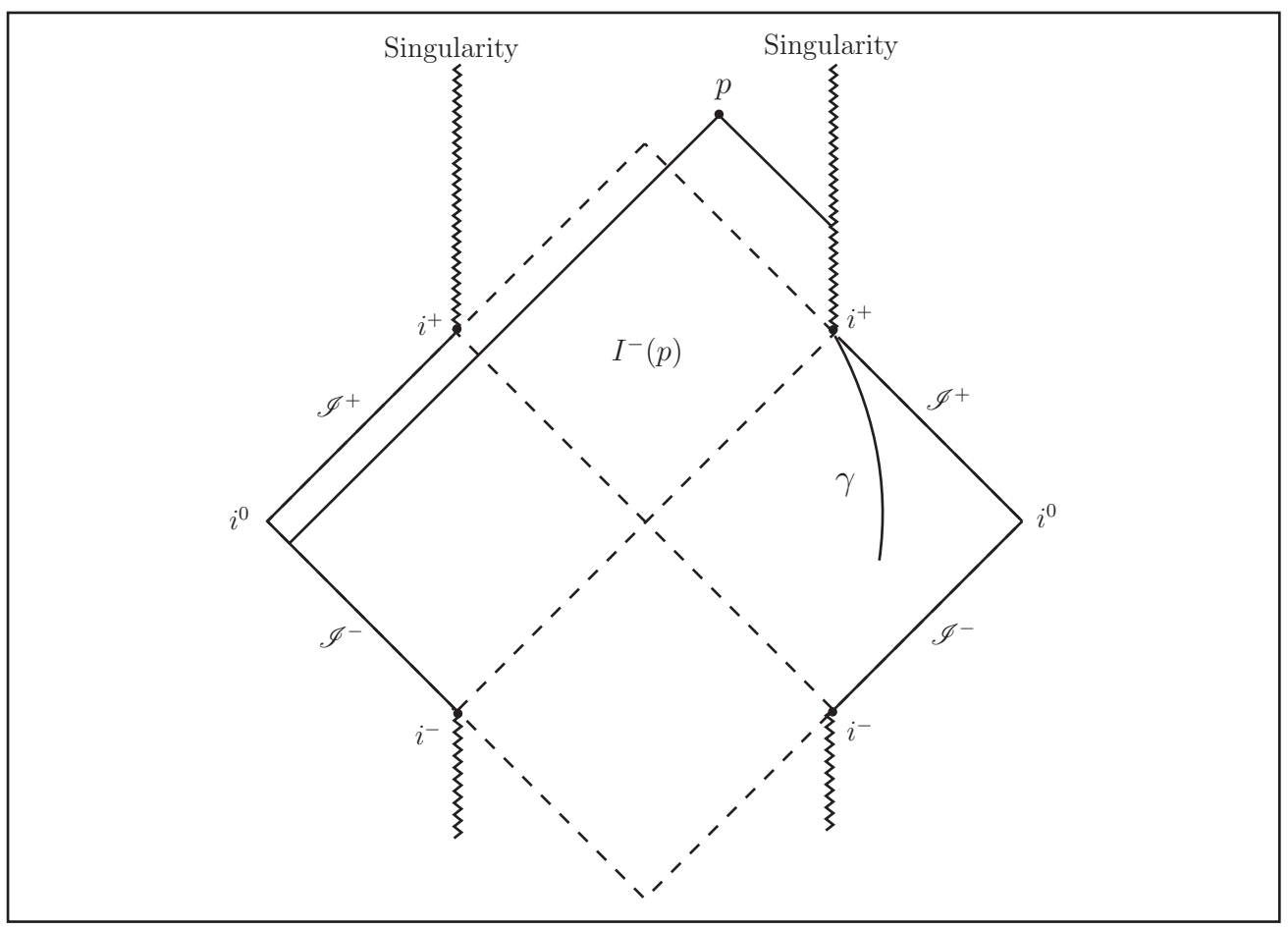

Figure 4: Conformal diagram of Reissner-Nordström spacetime.

Example. Reissner-Nordström spacetime. ${ }^{8}$ This spacetime represents a spherically symmetric electrically charged body (see the conformal diagram given in Figure 4). In the diagram, the future-directed timelike curve $\gamma$ approaches the singularity at $i^{+}$and is such that $\int_{\gamma} d \tau=\infty$. However, the point $p$ is such that $\gamma \subset I^{-}(p)$.

\section{An Example M-H Setup}

We have outlined seven conditions regarding the physicality of $\mathrm{M}-\mathrm{H}$ setups. These conditions seem to be substantial in that no M-H setup presented in the literature escapes them all. Accordingly, one might be tempted to conclude that there do not exist physically reasonable $\mathrm{M}-\mathrm{H}$ setups. However,

\footnotetext{
${ }^{8}$ Details can be found in [9, pp. 156-161].
} 
we suggest here that such a conclusion may be unwarranted. Indeed, we now present a spacetime which avoids all of the problems discussed above. In lieu of a formal existence proof of such a model, we offer an argument sketch accompanied with diagrams which, we hope, will better convey the basic structure of the example in mind.

Proposition. There exists an M-H setup $\left(\left(M, g_{a b}\right), p, \gamma\right)$ such that all of the following hold:

(i) $\left(M, g_{a b}\right)$ is inextendible.

(ii) $\left(M, g_{a b}\right)$ is stably causal.

(iii) $\left(\left(M, g_{a b}\right), p, \gamma\right)$ satisfies the bounded blueshift condition.

(iv) $\left(M, g_{a b}\right)$ satisfies the dominant and strong energy conditions.

(v) $\left(\left(M, g_{a b}\right), p, \gamma\right)$ satisfies the finite acceleration condition.

(vi) $\left(M, g_{a b}\right)$ satisfies the tidal force condition.

(vii) $\left(\left(M, g_{a b}\right), p, \gamma\right)$ satisfies the signal reliability condition.

Proof Sketch. Here, we provide a two-dimensional example. It is not difficult to see that the example generalizes to spacetimes of dimension four.

Let $\left(\mathbb{R}^{2}, \eta_{a b}\right)$ be Minkowski spacetime in standard $t, x$ coordinates (here $\left.\eta_{a b}=\nabla_{a} t \nabla_{b} t-\nabla_{a} x \nabla_{b} x\right)$. Now, cut slits $S_{i}, U_{i}, L_{i}$, and $C$ as shown in the bottom portion of Figure 5. For each $i \in \mathbb{N}$ identify the upper edge of $U_{i}$ with the lower edge of $L_{i+1}$ (excluding boundary points). Similarly, identify the lower edge of $U_{i}$ with the upper edge of $L_{i+1}$. Let $\left(M, \eta_{a b \mid M}\right)$ be the resulting spacetime.

Now consider another copy of Minkowski spacetime $\left(\mathbb{R}^{2}, \eta_{a b}\right)$. Now, cut slits $S_{i}^{\prime}$ and $C^{\prime}$ as shown in the top portion of Figure 5 . Let $\left(M^{\prime}, \eta_{a b \mid M^{\prime}}\right)$ be the resulting spacetime. Now we attach the spacetimes $\left(M, \eta_{a b \mid M}\right)$ and $\left(M^{\prime}, \eta_{a b \mid M^{\prime}}\right)$ as follows: For all $j \in \mathbb{Z}$, identify the upper edge of $S_{j}$ with the lower edge of $S_{j}^{\prime}$ (excluding boundary points). Similarly, identify the lower edge of $S_{j}$ with the upper edge of $S_{j}^{\prime}$. Make similar identifications with the sets $C$ and $C^{\prime}$. Let the resulting spacetime be $\left(M^{\prime \prime}, g_{a b}\right)$.

Let $p$ and $\gamma$ be as shown in Figure 6. Note that $\gamma$ continues until the lower edge of $U_{0}$ which, by construction, is identified with the upper edge of $L_{1}$. 
Similarly, the curve $\gamma$ continues until it reaches the lower edge of $U_{1}$ which is identified with the upper edge of $L_{2}$. The process continues ad infinitum. Thus, it is clear that $\int_{\gamma} d \tau=\infty$. It can be verified that there exists a future directed timelike curve from every point in $\gamma$ to the lower edge of $S_{j}$ for some $j$. So there is a future directed timelike curve from any point in $\gamma$ to the upper edge of $S_{j}^{\prime}$ for some $j$. But clearly, the lower edge of $C$, and hence the upper edge of $C^{\prime}$ is in $I^{-}(p)$. So, the upper edge of every $S_{j}$ is in $I^{-}(p)$. So, $\gamma \subset I^{-}(p)$ and $\left(M^{\prime \prime}, g_{a b}\right)$ is an M-H spacetime.

Now we show that $\left(\left(M^{\prime \prime}, g_{a b}\right), p, \gamma\right)$ satisfies conditions (i)-(vii). By construction, $\left(M^{\prime \prime}, g_{a b}\right)$ is inextendible and locally carries the Minkowski metric. So, (i), (iv), and (vi) are easily satisfied. Because the curve $\gamma$ is a geodesic, condition (v) is satisfied as well. Let $\Sigma$ be the spacelike hypersurface as shown in Figure 6. Every past-inextendible causal curve from $\gamma$ intersects $\Sigma$. So, $\gamma \subset D^{+}(\Sigma)$. One can also note that, for any point in $\Sigma$, there is a future-directed timelike curve from that point to some $S_{j}$ or to $p$. So, this implies that $\Sigma \subset I^{-}(p)$. Also, it an be easily verified that $p \in \operatorname{int}\left[D^{+}(\Sigma)\right]$. So, $\left(M^{\prime \prime}, g_{a b}\right)$ satisfies condition (vii).

Let $K_{i}$ and $\gamma^{\prime}$ be as shown in Figure 6. Without too much trouble, it is possible to verify that $\left(V^{\prime} a k_{a}\right) /\left(V^{a} k_{a}\right)=1$ where $V^{a}$ and $V^{\prime a}$ are, respectively, the unit tangent to $\gamma$ and $\gamma^{\prime}$ and $k^{a}$ is the tangent to the $K_{i}$. So $\left(\left(M^{\prime \prime}, g_{a b}\right), p, \gamma\right)$ satisfies condition (iii). Note also that the order of signal transmission matches the order of signal reception.

Finally, one can verify that $\left(M^{\prime \prime}, g_{a b}\right)$ is stably causal therefore satisfying condition (ii). Here, we simply show that it contains no closed timelike curves. We first claim that any future-directed timelike curve $\gamma_{s}$ with past endpoint $s \in M^{\prime}$ cannot return to $s$. It is easy to see that if $\gamma_{s}$ never leaves $M^{\prime}$, it cannot return to $s$. And if $\gamma_{s}$ does leave $M^{\prime}$, it must do so in one of two ways: either through one of the $S_{i}^{\prime}$ or through $C^{\prime}$. Clearly, if $\gamma$ leaves through $C^{\prime}$ or one of the $S_{i}^{\prime}$, and hence enters $M$, it can never leave $M$. So, there is no closed timelike curve through any point in $M^{\prime}$. Now consider any point $s \in M$. We also claim that any future-directed timelike curve $\gamma_{s}$ with past endpoint $s \in M$ cannot return to $s$. Because of the previous argument, such a curve cannot enter $C$ or any $S_{i}$ and be closed. If $\gamma_{s}$ enters some $L_{i}$, then it must exit some $U_{i}$ and can never return to any point to the past of any $L_{i}$ (including $s$ ). If $\gamma_{s}$ enters some $U_{i}$, it may exit through any $L_{j}$ where $i<j$. But, for all $i<j$ the timelike future of $L_{j}$ does not contain $U_{i}$. So, $\gamma_{s}$ cannot return to $s$. It is clear that any $\gamma_{s}$ which remains in $M$ but does not enter any $U_{i}$ or $L_{i}$ cannot be closed. 


\section{Conclusion}

We want to make it very clear that we do not feel that the example just presented is physically reasonable. We simply emphasize that the potential problems outlined above fail to rule it out. In addition, it must be remembered that the proposition can have physical relevance even if the particular cut and paste model constructed in the proof does not. Geroch explains:

"The space-times obtained by cutting and patching are not normally considered as serious models of our universe. However, the mere existence of a space-time having certain global features suggests that there are many models - some perhaps quite reaonable physically - with similar properties" [6, p. 78].

Of course, one can consider other requirements in addition to those presented here. Here, we briefly note some of these.

One way of ruling out our example above is to insist that spacetime be "hole-free" [7]. Intuitively, this condition guarantees that, for any spacelike surface $\Sigma$, the set $D^{+}(\Sigma)$ is "as large as it can be". We note here, however, that it is not clear that all physically reasonable spacetimes are hole-free [12], [14]. But, even granting this assumption, it is not as if a no-go theorem is then straightforwardly obtained.

A different sort of objection is hinted at by Earman and Norton. Following [15], they believe that although the sender, by construction, has "time enough" to complete its tasks, a physically reasonable $\mathrm{M}-\mathrm{H}$ setup must guarantee that there is "world enough" as well. The problem is that a computational device with a limited amount of computational space does not seem to have the resources to complete a supertask [3]. We acknowledge that this concern deserves attention, but for now, it is unclear how exactly to formalize such a worry.

Next, Norton ${ }^{9}$ has pointed out the possibility of energy related problems even for M-H setups satisfying the bounded blueshift condition. The concern is that if the receiver, in a finite time, receives an infinite number of signals from the sender and if each signal has the same energy on arrival, then the

\footnotetext{
${ }^{9}$ Private communication.
} 
total energy received may diverge. This may well be a serious problem. But, we also note that if the $\mathrm{M}-\mathrm{H}$ setup satisfies the signal reliability condition, the sender need not send an infinite number of signals to the receiver in order to complete certain supertasks - at most one is needed. Imagine the sender checking a conjecture of number theory for ' 1 ', for ' 2 ', etc. If a counterexample to the conjecture is found, the sender sends one signal to the receiver. Otherwise, no signal is ever sent. ${ }^{10}$ Since the signal (or non-signal as the case may be) is trustworthy in the sense of the signal reliability condition, the receiver would seem to be able to settle the conjecture.

Finally, one might insist that the receiver must "bring about" the supertask instead simply finding it already completed. Consider an $\mathrm{M}-\mathrm{H}$ setup $\left(\left(M, g_{a b}\right), p, \gamma\right)$. In order for a supertask is to be completed at $p$, then at some point $q$ (where $\gamma \cup\{p\} \subset I^{+}(q)$ ), the receiver must manufacture and initialize a computational device. But, it seems there would be no reason whatsoever for the receiver to manufacture and initialize the device at $q$ if there was no assurance at $q$ that a supertask at may be completed at $p$. In other words, it seems that part of what it means to say that the receiver brings about a supertask is that she is be able to predict, from $q$, the relevant spacetime structure to the causal future of $q .{ }^{11}$ And, given that $\left(M, g_{a b}\right)$ necessarily fails to be globally hyperbolic, the receiver seems to be incapable of such predictions. (We note, however, that there is a sense in which every observer in every spacetime is unable to make sure predictions concerning her casual future [13].)

This objection is certainly significant as a practical matter but we argue that it does not undermine the physical implementability of supertasks. It may be that that the receiver at $q$, not knowing with certainty the spacetime structure to her casual future, decides to manufacture and initialize a computational device anyway. She simply bets that at some later point $p$, the intended supertask may be completed. If her hopes are realized, wouldn't we want to say that she, in some way, brought about the outcome?

In sum, there are indeed other worries concerning the physical reasonableness of $\mathrm{M}-\mathrm{H}$ setups. But, given the resilience of examples like the one presented above, it is not yet clear that such potential problems will turn out to prohibit the physical implementability of supertasks.

\footnotetext{
${ }^{10}$ This setup is suggested in [3, p. 29].

${ }^{11}$ I am grateful to Robert Geroch for this point.
} 
Acknowledgments I am grateful to Robert Geroch, David Malament, John Norton, and an anonymous referee for reading an earlier draft and offering valuable suggestions. I also wish to thank John Earman as well as the Southern California Philosophy of Physics group for helpful discussions on this topic.

\section{References}

[1] Chakrabarti, S., Geroch, R., Liang, C.: Timelike curves of limited acceleration in general relativity. J. Math. Phys. 24, 597-598 (1983)

[2] Earman, J.: Bangs, Crunches, Whimpers, and Shrieks. Oxford University Press, Oxford (1995)

[3] Earman, J., Norton, J.: Forever is a day: supertasks in Pitowsky and Malament-Hogarth spacetimes. Philos. Sci. 60, 22-42 (1993)

[4] Earman, J., Norton, J.: Infinite pains: the trouble with supertasks. In: Stich, S., Morton, A. (eds.) Benacerraf and His Critics, pp. 231-261. Blackwell, New York (1996)

[5] Geroch, R.: Domain of dependence. J. Math. Phys. 11, 437-449 (1970)

[6] Geroch, R.: Spacetime structure from a global viewpoint. In: Sachs, B. K. (ed.) General Relativity and Cosmology, pp. 71-103. Academic Press, New York (1971)

[7] Geroch, R.: Prediction in general relativity. In: Earman, J., Glymour, C., Stachel, J. (eds.) Foundations of Space-Time Theories. Minnesota Studies in the Philosophy of Science, vol. 8, pp. 81-93. University of Minnesota Press, Minneapolis (1977)

[8] Geroch, R., Horowitz, G.: Global structure of spacetimes. In: Hawking, S., Israel, W. (eds.) General Relativity: An Einstein Centenary Survey, pp. 212-293. Cambridge University Press, Cambridge (1979)

[9] Hawking, S. W., Ellis, G. F. R.: The Large Scale Structure of SpaceTime. Cambridge University Press, Cambridge (1973) 
[10] Hogarth, M.: Does general relativity allow an observer to view an eternity in a finite time? Found. Phys. Lett. 5, 173-181 (1992)

[11] Hogarth, M.: Non-Turing computers and non-Turing computability. In: Hull, D., Forbes, M., Burian R. (eds.) Proceedings of the Biennial Meeting of the Philosophy of Science Association 1994, pp. 126-138. University of Chicago Press (1994)

[12] Krasnikov, S.: Even the Minkowski space is holed. Phys. Rev. D 79, 124041 (2009)

[13] Manchak, J.: Is prediction possible in general relativity? Found. Phys. 38, 317-321 (2008)

[14] Manchak, J.: Is spacetime hole-free? Gen. Relativ. Gravit. 41, 1639$1643(2009)$

[15] Pitowski, I.: The physical Church thesis and physical computational complexity. Iyyun 39, 81-99 (1990)

[16] Wald, R. M.: General Relativity. University of Chicago Press, Chicago (1984) 

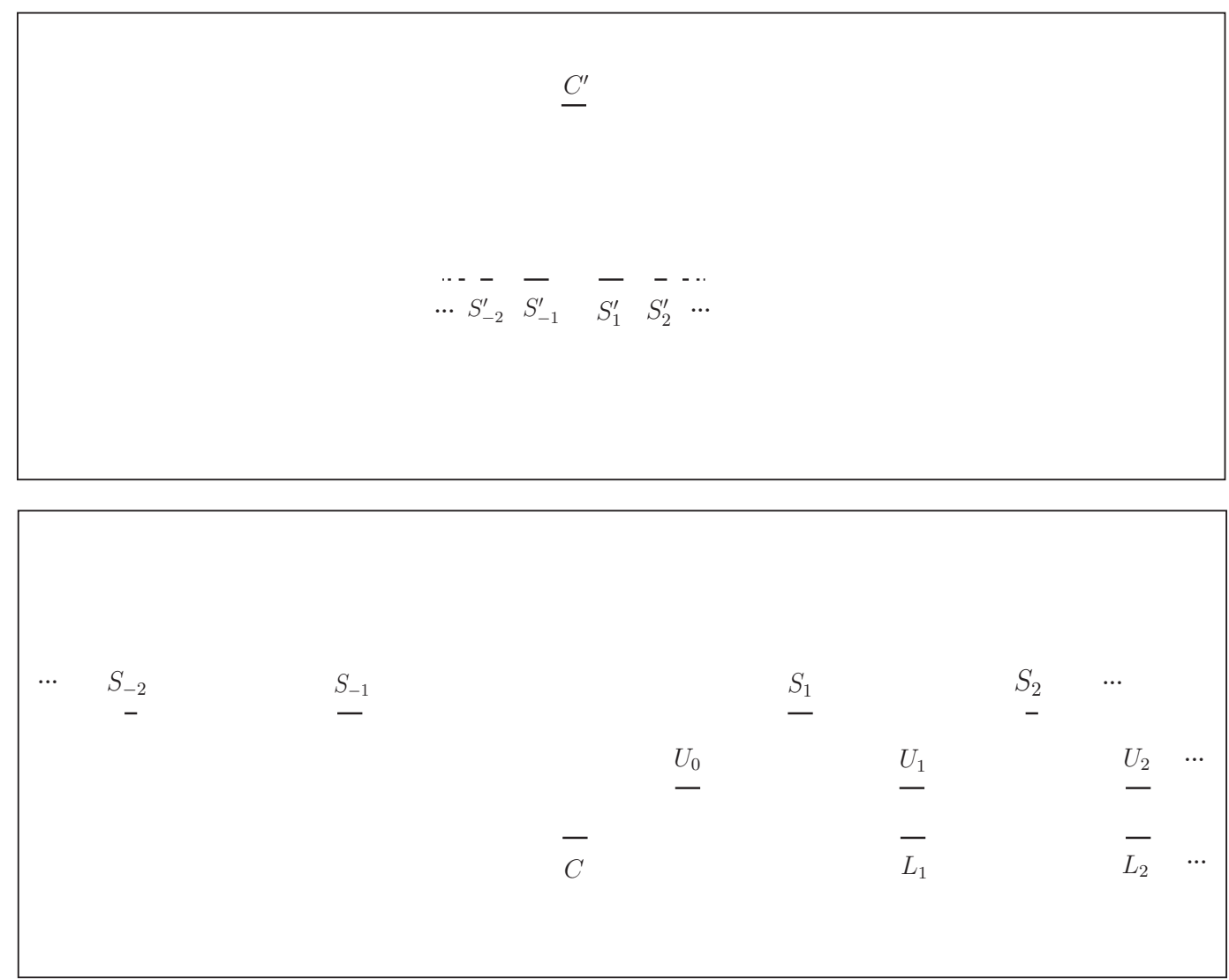

Figure 5: The spacetime above is $\left(M^{\prime}, \eta_{a b \mid M^{\prime}}^{\prime}\right)$. The spacetime below is $\left(M, \eta_{a b \mid M}\right)$ where, for each $i \in \mathbb{N}$, the upper edge of $U_{i}$ is identified with the lower edge of $L_{i+1}$ and vice versa. The two spacetimes are connected to make $\left(M^{\prime \prime}, g_{a b}\right)$ by making the following identifications: For all $j \in \mathbb{Z}$, identify the upper edge of $S_{j}$ with the lower edge of $S_{j}^{\prime}$ and vice versa. Make similar identifications with the sets $C$ and $C^{\prime}$. Drawn to scale. 



Figure 6: The spacetime $\left(M^{\prime \prime}, g_{a b}\right)$ with $p, \gamma, \gamma^{\prime}, \Sigma$, and $K_{i}$ indicated. Drawn to scale. 